\title{
Purposing AAL to an Aging Population: AAW (Walking), AAD (Driving) and the IoV (Internet of Vehicles)
}

\author{
Ciarán Mc Goldrick, SMIEEE, R. Vince Rabsatt and Mario Gerla, FIEEE
}

\begin{abstract}
The Internet of Things is construed as a broad interconnectivity of everyday devices and entities, such that their collaborative engagements can create superior externalities. It is distinguished from the "sensorweb" through the capabilities of IoT devices to operate and reason on, and with, inputs from multiple sources. In this work we postulate that vehicles have a previously unexplored role to play in driving widespread acceptance, and adoption, of mobile cloud, IoT and AAL principles, concepts and systems. We argue that, historically, deployment of new, disruptive technological paradigms has been slow and the notion of IoT devices being deeply embedded in our everyday environment, and working and collaborating to enhance and support our daily existences (Ambient Assisted Living), makes "realizing tomorrows potential difficult. But not impossible" [1] for industry actors. This work re-envisions common approaches to presenting AAL research and focuses on emerging and future enabler technologies and platforms that will provide direct AAL interactions and experiences for end users. The use-case and deployment scenario is that of present day and future vehicular and transportation infrastructure networks, with exemplars in the global societal challenge of healthcare for aging populations.
\end{abstract}

Index Terms-Ambient Assisted Living, Internet of Vehicles, Ambient Assisted Driving, Ambient Assisted Walking, AAW, AAD, Autonomous Driving, Aging, Elderly, Independent Living.

\section{INTRODUCTION}

A MBIENT Assisted Living (AAL) is often perceived as combining the synergy of smart home and smart phone scale technologies, with application scenarios such as reducing patient care costs. Smart phones allow the accurate monitoring of human activities and movements in the home and can provide wellness and vital signs monitoring, especially if coupled with embedded body sensors. Smart home sensors/actuators close this loop and assist in monitoring (e.g. patient positioning, fall detection/prevention, etc) and in making the environment comfortable for the patient (e.g. adjusting temperature, light, sound, etc).

In this paper, we project AAL principles, deployments and use-cases into the vehicular networking and control domain, and demonstrate how vehicles can be a translational nexus for widespread integration and adoption of AAL technologies. A key differentiator in this work is the notion of extending AAL concepts to motive scenarios e.g. for assisting patients $\mathrm{ON}$

C. Mc Goldrick is with the School of Computer Science and Statistics, Trinity College Dublin, e-mail: (Ciaran.McGoldrick@scss.tcd.ie).

R. Rabsatt and M. Gerla are with the Department of Computer Science, University of California, Los Angeles, e-mail: (\{rrabsatt, gerla\}@cs.ucla.edu).
THE MOVE. This focus is very important for the future of our society because there are many active elderly that could still walk and drive, but today are home bound because of the risk they represent to themselves and others. As many of these citizens continue to move around and drive without assistance, the resulting incidents increase healthcare and insurance costs for all [2]. Thus AAL for active, mobile aging populations, e.g. Ambient Assisted Walking (AAW) and Ambient Assisted Driving (AAD), must be advanced for solving compelling social problems. Fortunately, technological advances are working in our favor. We consider two AAL scenarios involving active, mobile patients a practical enduser application targeting reductions in skin cancer and the protection of sensitive skin amongst both active and aging walkers (the UV-Guardian Phone), and an integrated systems level approach exploiting vehicular networking solutions that incorporates the safe navigation of problem drivers through autonomous vehicle technologies. The UV Guardian is an example of a present day AAL solution in the domain of Ambient Assisted Walking (AAW), whilst the more extensive vehicular platform descriptor extrapolates ongoing research into future AAL and AAD solutions that have practical applications for aging population demographics. The examples highlights the Ambient and Assistive solutions developed and deployed, and the extended scope of the vehicular AAD platform solution is expounded for healthcare in both urban and rural scenarios.

\section{RELATED WORK}

The aging population demographics in many developed economies means that Ambient Assisted Living has received considerable interest from both the research community and industries alike. As aging and elderly people seek to remain independent, active and resident in their own homes for as long as possible[3], AAL can help create and sustain supportive, interactive environments where individuals interact with intelligent devices that are capable of monitoring and responding to their immediate needs.

A number of studies have focused on applying AAL principles to homes. Aware Home [4] created a living laboratory in a home using ubiquitous computing technologies in order to be aware of the whereabouts and activities of the occupants. Exploring the balance between the simpler life that AAL provides and the complexity of managing the technology is considered in [5], where they examined the appropriate technologies, applications and social impact of ambient intelligence in the 
home. In [6] telemonitoring processes are employed where Internet of Things (IoT) facilitates remote home monitoring of patients by physicians.

Modern smartphones are the most common AAL interaction device that individuals encounter. Their widespread usage, and global spread, make them compelling AAL interaction devices - both in terms of capabilities and scale of the userbase. The UV Guardian uses Android phones [7] to predict a pedestrian's (AAW) potential ultra-violet exposure and to provide guidance and recommendations on avoiding overexposure. Mobile Habits [8] uses wireless position strategies to estimate a users outdoor activities. In [9] body sensors are used to create a wearable system that is coupled with a smart phone to estimate a users energy expenditure. These strategies provide additional context awareness of the type that will be instrumental in personalised AAL and AAW solutions.

Our work incorporates principles and concepts akin to those espoused in the above works, and extends them to future vehicular environments and beyond.

\section{UV GUARDIAN}

UV Guardian[7] is an early Ambient Assistive Walking (AAW) system developed in the Networks Research Laboratory in UCLA. It leverages many of the primitives that underpin subsequent and recent AAL research, applications and solutions - local information, 3/4G communications, and centralised cloud processing and analysis.

UV Guardian tracks and aggregates the user's exposure to ultraviolet (UV) Rays based on their outdoor time and activity. As over-exposure to UV wavelengths can lead to severe skin damage, accurate exposure knowledge helps both end-users and medical professionals to assess and ameliorate long-term impacts. A regular Android phone running the UV Guardian App estimates a person's UV exposure as they engage in outdoor activities in an urban environment.

UV Guardian utilizes participatory sensing to construct a real-time UV irradiance map as a function of time relative to the Sun's position. Mobile phones connect to UV sensors via Bluetooth to collect UV irradiance data, which is periodically uploaded along with the sampled GPS location and a timestamp. Pedestrians can use UV Guardian to identify comparable walking paths that minimize UV exposure.

Supportive AAL solutions, such as UV Guardian, are of particular significance for optimising healthcare and longevity outcomes for both active and aging populations. Younger, active people are at greater risk of skin damage due to outdoor pursuits such as running, cycling, etc. This population segment has a near symbiotic relationship with their smart phones so UV guardian is a valuable advisory tool on an ongoing basis. As individuals age their faculties become increasingly compromised causing them to pay less attention to risk factors in their environments. They are at increased risk of getting lost or of injuring themselves by falling. The capabilities of AAL solutions, such as UV Guardian, to track a patients position and activity can be used to closely monitor and maximise outcomes for elderly users. Sensor information (e.g. location, accelerometer data, etc.) can be aggregated with other AAL data and analysed (in the cloud) to not only provide sunscreen recommendations but also fine-grained recommendations and guidance for caregivers.

As a precursor to a more detailed exposition of what vehicular networking will bring to motive AAL and AAD scenarios, we briefly explore the roles and capabilities that autonomous vehicles and vehicular networking can provide an end-user outdoor mobility/AAL application like UV Guardian.

A significant contributor to vehicular related fatalities, particularly amongst the aging and infirm, is that of pedestrian impact e.g. at pedestrian crossings. Pedestrians are usually alerted by the noise of the approaching vehicle. Those with impaired hearing e.g. from aging or trauma, cannot detect that signal. Moreover, electric vehicles make virtually no distinguishable noise. AAL/AAD aware vehicles may use their forward facing cameras and IR transmission and detection arrays to scan the road. A pedestrian whose garments detect and react to this infrared beaconing can be automatically alerted through their smart phone ringing to the potential danger. In this case, the smart phone monitors the walk and can correlate the fact that the pedestrian will step in the road with the beaconing signal from oncoming cars. A complementary protection approach is that of using an ultrasound beacon sent by pedestrians BEFORE they step in the road and picked up by the oncoming cars and/or emitted from the vehicles front ultrasonic parking sensors and received by the pedestrians embedded receiver.

The above AAW applications are quite different but all share a common premise the correlation of activity information maintained by the pedestrians smart phone (eg, position, acceleration, etc) with environmental signalling, like UV information or infrared car beacons. It is clear that this represents a major departure from the indoor AAL mode of operation. In the intelligent home, for example, the ambient learns about the patient, it can anticipate movement, change lighting and thermostat settings, etc. In other words, the intelligent environment observes the patient and tells it what to do. By contrast, in the outdoor mobility model, the individuals body network gateway device (commonly a smartphone) plays the dominant role in integrating and actuating pedestrian actions and warnings. This inversion of the traditional AAL model is expected of course, as the outdoor environment is not familiar with the individual and does not keep a model of him/her. Moreover their movement patterns will not be consistently predictable so AAL systems must now accommodate, integrate and exploit dynamic movement and mobility scenarios.

Interestingly, as one moves from the AAW to the AAD scenario, one finds that the two aspects (indoor and outdoor) converge. The intelligent home becomes the intelligent cockpit and cabin with the smart phone and/or car router fusing the driver activity and (health) state information with the signals from the external environment (vehicular grid/mobile cloud).

\section{Future Vehicular Platforms}

A number of common themes underpin the evolution of transportation systems, including those of efficiency, reliability, throughput and innovation. Vehicular platforms and systems provide compelling, large-scale infrastructures through 
which society and its citizens can rapidly embrace many of the benefits of IoT and AAL deployments. In this work we articulate an integral role for future innovative vehicular platforms in the context of form, interconnectivity and capabilities in a converged IoT/AAL future. Our exemplars will be those of aging and elderly population groupings in i) large, industrialised urban landscapes e.g. Los Angeles, and ii) those in more remote rural communities e.g. rural Ireland, and the AAL benefits that future secure, autonomous vehicular platforms can offer an aging population demographic [10].

\section{A. Physical forms}

Vehicles are designed and manufactured to leverage and exploit the physical properties and constraints of the carrier medium - in general wheeled and tracked vehicles operate on the earth; buoyant, displacement and submergent vessels on/in water; and aerodynamic propeller or jet powered vessels in the air. New technologies and approaches e.g. levitation [11] will further enhance the efficiency, reliability and throughput in these domains, but the basic carrier mediums of land, air and water will predominate in the foreseeable future. Innovation in transportation form and function is largely being driven by the increasingly interconnected nature of both vehicles and the transportation infrastructure. A key area of research and innovation is that of autonomous vehicles. Autonomous vehicles not only help empower aging, infirm and impaired citizens by restoring their capability for safe, independent travel, but they also provide a powerful, non-invasive, nonthreatening modality by which to monitor, interact with, and respond to their passengers state of being.

Autonomous vehicles must be capable of operating entirely independently of a human passenger or operator. They must respect national laws, safety requirements and social conventions in their activities. They should be capable of independently replenishing their energy source, and should be able to appropriately react to evolving situations. For empowering and enabling an aging population we expect autonomous vehicles to, at a minimum, i) be able to safely operate in all driver use case scenarios and ii) safely operate in driverless mode. For maximum utility they should be able to operate both independently of, and in collaboration with, other vehicles and services. In rural areas the independent mode of operation might be most common, although not exclusively so. In urban areas the collaborative mode of assistive operation will predominate. All autonomous vehicles will have sensor arrays - both externally and internally. The external sensors will provide the primary control inputs for the vehicle - through radio, visual, acoustic and light signalling. The internal sensor arrays will integrate capabilities of benefit to all passengers, but of particular assistive significance for an aging population. As nanoscale, body and personal area networks gain traction in the context of the Internet of Things, and automated and implantable healthcare devices become commonplace, the vehicle becomes an increasingly significant actor in both the communication and AAL chains. The vehicle provides power for wireless charging of embedded body devices, can act as a trusted interrogator and aggregator of embedded and wearable device data, can function as an in-situ diagnostic agent (using seat embedded sensors, cameras and audio prompting via the entertainment system) and can provide alerting to emergency personnel as necessary.

The societal and AAL potential of Autonomous Vehicles becomes more compelling when considered in the context of the more widespread deployment and integration of IoT technologies. Consider autonomous ambulance/medivac units which are locally based, or located where medical services are sparse. Initial responder protocols and diagnostic procedures can be effected in the vehicle and remotely advised to the local medical center. Follow up appointments and procedures can be instantly scheduled or the patient can be immediately transported to the nearest hospital as required. In tandem, other autonomous ambulances in the wider locality can be dynamically relocated in order to satisfy overall service provision guarantees across the region.

\section{B. Connectivity}

Present day assistive living systems commonly leverage 3 and 4G cellular communication technologies to connect systems and devices to centralised control infrastructures often cloud based solutions. These approaches currently work well in urban areas, where there is a high density of cellular infrastructure deployed. However their performance and availability degrades rapidly as they travel into less populated areas where the communications infrastructure is sparser. In extreme cases there may be no traditional communications infrastructure e.g. offshore, in deep valleys, at high altitudes, in disaster scenarios. Reliable functionality in these "outage" scenarios necessitates careful system design at the outset, but potential solution paths that are being developed and advanced in a transportation context have strong resonance for IoT embedded communication scenarios also.

- A key driver of current $5 \mathrm{G}$ activities is to scalably accommodate mobile device interconnectivity and mobility on a global (IoT) scale. New hardware, architectures, protocols and technologies will be necessary to fully realise a unified system that provides these capabilities. 5G systems must accommodate seamless integration with both existing and future communication modalities e.g. wireless handoff, P2P integration, whilst also accommodating the dynamic mobility, energy saving and disconnection behaviours that will characterise many IoT and AAL devices. As a corollary to this connectivity metaphor, there will be many scenarios where IoT devices cannot, or will not, (regularly) connect to $5 \mathrm{G}$ networks. Devices may be designed to conserve energy and sleep for extended periods, awakening according to a duty cycle schedule or in response to a specific external actuation. Devices may elect not to connect to insecure networks, or to communicate with untrusted or unrecognised networks. Devices may be deployed in disaster scenarios and need to dynamically discover neighbours and communication routes to control and actuation nodes. All these capabilities depend on reliable, secure, highly available wireless communications capabilities at proximate, local, national and transnational scales, but also on the seamless integration of fixed and mobile 
devices across the many different communication complexities and device scales that form the Internet of Things. Future vehicular networks provide a compelling connectivity solution in these scenarios. The platforms will support multiple communication technologies - Wifi, BLE, 5G, V2V, and will exploit both connection-oriented and connection-less e.g. P2P, interactions. Networks of vehicular devices will form vehicular clouds for data aggregation, decision taking and dissemination purposes. The vehicular cloud forms a subset of the more general mobile cloud metaphor, but is markedly more powerful in terms of individual device capabilities and availability. Moreover society is adjusted to, and accepting of, the notion of electronic systems and devices constructively assisting people (on a daily basis) on their travels - thus vehicles and transportation infrastructures provide a natural first-adopter platform from which to induce broader acceptance of, and engagement with, global scale AAL platforms and systems.

\section{Capabilities}

Many of the active research themes in the AAL domain have direct corollaries in the autonomous vehicle research space - mobility solutions, security, distributed intelligence, mobile cloud, e-health, etc. Thus vehicular networking and autonomous vehicle solutions are significant to IoT and AAL researchers in ways beyond simply providing practical realworld evaluation and deployment platforms. The remainder of this paper will focus on two complementary technologies that underpin autonomous driving - those of cooperative adaptive cruise control(CACC), and vehicular platooning. Both systems are complementary, can operate in both centralised and decentralised 'local' P2P modes, leverage the vehicular cloud, prioritize security, and integrate inputs from a variety of different systems in forming an actuation decision. Thus they capture many of the key facets of AAL research and systems design challenges. Both CACC and platooning are viewed as key assistive technologies for all drivers, but particularly for aging populations where alertness and responsivity may be compromised or impaired.

\section{ENABLING VEHICULAR RESEARCH}

\section{A. Autonomous Driving}

In urban environments it is common for drivers to access a highway in order to reach their destination, and in this environment a large number of vehicles utilizing the highway at peak times will likely lead to traffic congestion [12]. High utilization potentially poses a challenge to elderly drivers who may face vision changes or declining motor skills and reflexes due to the natural aging process [13]. In the case of autonomous vehicles, if highway throughput is not managed properly, traffic efficiency can suffer. Platooning is seen as a promising application that facilitates travel in groups of vehicles, at relatively small inter-vehicular distances, all autonomously following their leading vehicle Vehicular platooning offers reduced fuel consumption, increased safety, and improved traffic capacity, leading to shorter travel times and less traffic congestion [14] - all of benefit to aging and increasingly infirm populations.
The coordination of a platoon relies on a vehicles ability to obtain downstream information from other vehicles and a control system to regulate the distance between vehicles and maintain stabilization. Vehicles equipped exclusively with radar use Adaptive Cruise Control (ACC) to sense an immediate neighbors position and speed. However, when coupled with wireless communication, Cooperative Adaptive Cruise Control can be achieved. CACC allows a vehicle to receive information such as acceleration from the platoon leader and the immediate preceding vehicle, which further reduces the inter-vehicle distance required [15]. Fig 2 shows the difference between ACC and CACC.

Fig 1 reports the results of an experiment where the lead car moves erratically varying its speed by $10 \mathrm{kph}$ in $5 \mathrm{~s}$. Fig. 1(a) shows the velocity of the cars in the platoon when headway between cars is $\mathrm{T}=0.3 \mathrm{~s}$. The velocity of the last car is totally out of phase suggesting that a crash may be imminent. Increasing the headway time (to $\mathrm{T}=1.2 \mathrm{~s}$ ) as shown in Fig. 1(b), smoothens out the oscillations and stabilizes the system, but it increases the gap from $10 \mathrm{~m}$ to $30 \mathrm{~m}$, neutralizing the benefits of platooning. Surprisingly, CACC can maintain perfect stability with just $5 \mathrm{~m}$ gap that is independent of speed. These results show that without CACC there is no platooning.

The leading vehicle of a platoon can be controlled by a human driver [16], a central entity via the Internet, such as an advanced traffic management system (ATMS) [17], or a locally mediated agreement between participating vehicles and infrastructure. The central entity can manage the selection of platoon leaders and provide acceleration patterns. Advances in $5 \mathrm{G}$ network technologies will make this a feasible option, given its design goal of accommodating high traffic volumes, providing increased system capacity, and provisioning for seamless and ubiquitous communication [18]. Intra-platoon dynamics will continue to exploit vehicle to vehicle (V2V) communication via dedicated short range communication (DSRC). In this system the central entity will have coarse-grain control over multiple platoons, leaving fine-grain control to the platoon leader. Advances in Internet of Things (IoT) deployments will also contribute to the quality of information obtained by the system. Roads will be outfitted with tags and sensors to send useful information to traffic control sites, and vehicles and roadside infrastructure will assist in collaborative decision making and autonomous actuation in both centralized and local contexts [19]. Whilst autonomous driving and coordinated platooning offer many compelling benefits in future transport modalities, it is important to note that they do not account for unforeseen eventualities, such as accidents, breakdowns, etc., in normal operation. The systems can respond dynamically and safely to such eventualities e.g by braking or taking avoiding action, but at the cost of introducing a delay or slowdown in the vehicular flow. In heavy traffic conditions this can give rise to traffic shockwaves, which radiate back from the site of the original incident and, indeed, can give rise to subsequent accidents and incidents.

\section{B. Managing and preventing Traffic Shockwaves}

Shockwaves can be particularly problematic for senior and impaired drivers, whose responsiveness and reaction times can 


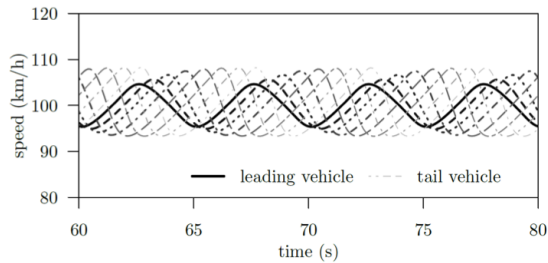

(a) ACC: headway $\mathrm{T}=0.3 \mathrm{~s}$

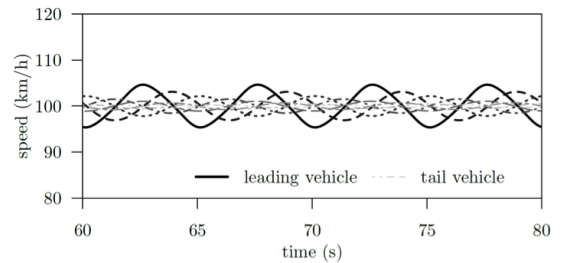

(b) ACC: headway $\mathrm{T}=1.2 \mathrm{~s}$

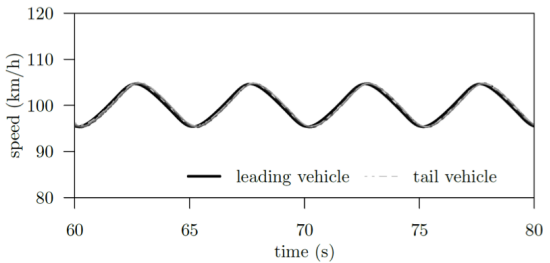

(c) CACC: radar + wireless communication

Fig. 1: Controller Comparison

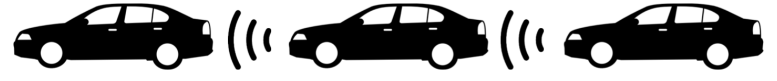

(a) ACC: radar (or lidar) based

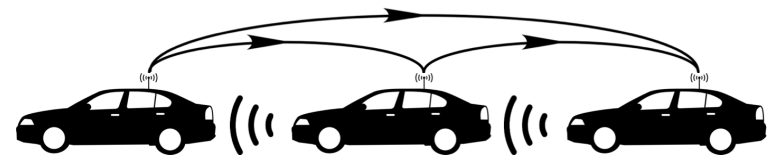

(b) CACC: radar + wireless communication

Fig. 2: Platoon Control System

be impaired. Fortunately modern V2V technology can help ensure their safety and wellbeing, and that of fellow road users, thereby empowering them to remain active and mobile in their later years. A primary cause of shock waves is the combination of high traffic demand, unexpected driver actions and physical perturbations such as ramps, construction sites or lane reduction.

Fig. 3 demonstrates the formation of a shock wave in a simple one-lane road scenario. Stop-and-Go traffic often seems to appear out of nowhere and the resulting congestions are often referred to as phantom jams. As one can see from Fig. 3 , vehicles are not able to react until the incident that triggered the wave is in line of sight of the driver. This results in hard braking manoeuvres in order to avoid a crash. Immediately afterwards the motorists accelerate again when leaving the congested area. It is clear that, for less alert or responsive drivers, such scenarios increase the potential for accident.

To help in this situation, many Advanced Driver Assistance Systems (ADAS) are implemented in luxury class vehicles. An example of such a system is the Adaptive Cruise Control System (ACC) that can maintain minimum safety headway to the vehicle ahead. Such systems can react much more quickly than a human driver to abrupt downstream vehicle manoeuvres, and can thereby operate effectively with much smaller safety distances. The wider effectiveness of such systems is curtailed by the Line of Sight constraint we previously identified.

To overcome the line of sight limitation and effectively mitigate shockwaves, a new communication protocol - Density Redistribution through Intelligent Velocity Estimation (DRIVE) was developed in [12]. A new Vehicle to Vehicle (V2V) communication protocol(DRIVE) is introduced to extend the awareness horizon far beyond the line of sight. With DRIVE it is possible to anticipate velocities upstream in the traffic flow in order to prevent congestions. A key novelty of DRIVE is

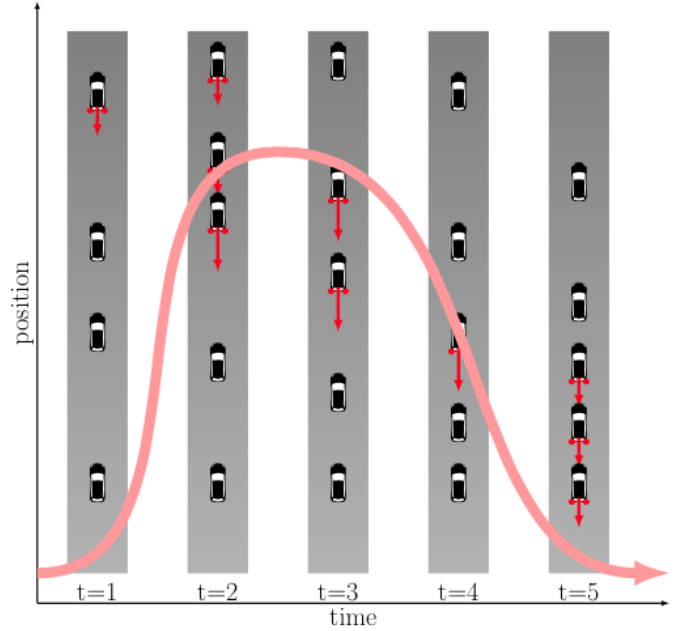

Fig. 3: Formation of a shockwave stream upwards

the approach proposed for estimating the traffic conditions in the interspace between two communicating vehicles without knowing the percentage of participants [12]. The DRIVE protocol is fully compliant with the IEEE 802.11p standards. Message propagation can be realized by the use of the Cooperative Awareness Message. DRIVE is a connectionless and event driven networking protocol, meaning that vehicles broadcast their messages in a burst, only in case of a significant slowdown or if the velocity falls below a given threshold. Each message has a certain Time To Live (TTL) to ensure that it reaches the neighbors that are within transmission range. The aim of the protocol is to redistribute the upstream vehicular density in a way that avoids the formation of a shock wave in case of a temporary peak in traffic demand.

When a slowdown event is detected, the leading vehicle sends a message containing the actual location and vehicle dynamics. The next vehicle in the information chain receives this message, adapts its velocity according to the recommendation by DRIVE, and sends a notification containing its own slowdown information further upstream rebroadcasting the received message. This chain of messages ends if the system determines that no action has to be taken, there is no vehicle within transmission range to receive a message or if the maximum propagation radius for a distinct message is reached. Simulation and small scale testbed results have shown that even a relative small percentage of DRIVE equipped cars (say 20 to $30 \%$ ) can dramatically reduce the shockwave occurrence 
[12]. Again, this is a clear example of 5G technology (as a reliable carrier for $\mathrm{V} 2 \mathrm{~V}$ messaging) helping make the driving experience safe and comfortable for the elderly. It is also an effective example of an adaptive ambient messaging and decision making behaviour occurring both across and within a grouping of vehicles.

\section{Autonomous Vehicles as A NeXus for AAL INTEGRATION}

The societal potential of Autonomous Vehicles becomes more compelling when considered in the context of the more widespread deployment and integration of Internet of Things(IoT) and Ambient Assistive Living(AAL) technologies. Much has been written on Personal Health, Body Area and Pervasive Health Networks in a connected AAL future (either centrally mediated or via opportunistic, P2P communications). A common example is where an individual's body area network can, in consultation with an expert system, provide early warning of a medical crisis. Consider the vehicular and intelligent transportation infrastructure role in such an AAL example: autonomous ambulance/medivac units which are locally based, or located where medical services are sparse. Initial responder protocols and diagnostic procedures can be effected in the vehicle and remotely advised to the local medical center. Followup appointments and procedures can be instantly scheduled or the patient can be immediately transported to the nearest hospital or medical facility as required. In life-threatening or time-critical situations e.g. organ transplant cases, traffic prioritisation measures can be instantaneously actioned and evolved; contra-flow lanes can be dynamically repurposed, platoons can be merged, junction signalling can be reprioritised, etc. In tandem, other autonomous ambulances in the wider locality can be dynamically relocated in order to satisfy overall service provision guarantees across the region.

There is however a dark side in autonomously driven vehicles - that of habituation to be driven and a consequential inability to react promptly in case of unforeseen events that necessitate prompt manual intervention. Consider for example a vehicular platoon. If an external attacker compromises the AAD systems of some of the cars in the platoon, it can easily cause incredible havoc by forcing incorrect behaviour and wrong responses in these cars. The honest, non-compromised cars will detect the anomalous behavior and will alert and advise drivers to take manual control immediately. This will pose a problem for infirm and medically impaired drivers. They may not be able to effect manual control promptly enough and, in the worst case, may bring the entire platoon down (e.g. accident). This observation suggests that some level of responsiveness constraint may be locally required from all drivers. It is envisaged that this will form an element of the dynamic passenger health assessment referred to earlier. Clearly AAL systems will also play a key role in this dynamic AAD assessment also.

\section{CONCLUSION}

In this work we argue that emerging and future vehicular networks will have a seminal role in driving widespread acceptance and adoption of AAL concepts and principles by and for mobile and outdoor citizens. The global challenge of improving healthcare and outcomes for aging population demographics is adopted as an exemplar. We describe a representative AAL mobile application (UV Guardian) and contrast it with home-based AAL solutions. We highlight how the introduction of a vehicular platform, and its associated mobility, can be used to further enhance and support a mobile users activities. We then extend the independent, aging exemplar to AAD - collaborative Ambient Autonomous Driving. Recent research advances in the domain are presented and solutions described. Some outstanding challenges for AAD are highlighted, as is the importance of proposed developments in $5 \mathrm{G}$, adhoc and bluetooth communications for AAL as a whole.

\section{REFERENCES}

[1] Ambient Computing : Putting the Internet of Things to work. [Online]. Available: http://dupress.com/articles/tech-trends-2015ambient-computing/

[2] Older drivers. [Online]. Available: http://www.iii.org/issue-update/olderdrivers

[3] L. Buys et al., "Research: Issues of active ageing: Perceptions of older people with lifelong intellectual disability," Australasian Journal on Ageing, vol. 27, no. 2, pp. 67-71, 2008.

[4] G. D. Abowd et al., "The aware home: A living laboratory for technologies for successful aging," in Proceedings of the AAAI-O2 Workshop Automation as Caregiver, 2002, pp. 1-7.

[5] M. Friedewald et al., "Perspectives of ambient intelligence in the home environment," Telematics and informatics, vol. 22, no. 3, pp. 221-238.

[6] A. Dohr et al., "The internet of things for ambient assisted living," in Information Technology: New Generations (ITNG), 2010 Seventh International Conference on, April 2010, pp. 804-809.

[7] J. Matthews et al., "Ultraviolet guardian-real time ultraviolet monitoring," Computer Science Department, University of California, Los Angeles, CA, 2012.

[8] A. Papliatseyeu and O. Mayora, "Mobile habits: Inferring and predicting user activities with a location-aware smartphone," in 3rd Symposium of Ubiquitous Computing and Ambient Intelligence 2008, pp. 343-352.

[9] H. Kalantarian et al., "Multimodal energy expenditure calculation for pervasive health: A data fusion model using wearable sensors," in Pervasive Computing and Communications Workshops (PERCOM Workshops),IEEE Int. Conf. on, March 2013, pp. 676-681.

[10] S. Kolski et al., Secure autonomous driving in dynamic environments: From object detection to safe driving. Citeseer, 2007.

[11] Slide. [Online]. Available: http://www.lexusint.com/amazinginmotion/slide/

[12] M. Forster, R. Frank, M. Gerla, and T. Engel, "A cooperative advanced driver assistance system to mitigate vehicular traffic shock waves," in INFOCOM, 2014 Proceedings IEEE, April 2014, pp. 1968-1976.

[13] J. Zhang et al., "Factors affecting the severity of motor vehicle traffic crashes involving elderly drivers in ontario," Accident Analysis \& Prevention, vol. 32, no. 1, pp. 117-125, 2000.

[14] M. Segata et al., "Towards inter-vehicle communication strategies for platooning support," in Communication Technologies for Vehicles (Nets4Cars), 2014 7th Int Workshop on. IEEE, 2014, pp. 1-6.

[15] R. Rajamani, Vehicle dynamics and control. Springer Science \& Business Media, 2011.

[16] C. Bergenhem et al., "Challenges of platooning on public motorways," in 17th World Congress on Intelligent Transport Sys, 2010, pp. 1-12.

[17] P. Fernandes and U. Nunes, "Platooning with ivc-enabled autonomous vehicles: Strategies to mitigate communication delays, improve safety and traffic flow," Intelligent Transportation Systems, IEEE Transactions on, vol. 13, no. 1, pp. 91-106, 2012.

[18] F. Haider et al., "Cellular architecture and key technologies for $5 \mathrm{~g}$ wireless communication networks," IEEE Comms Magazine, p. 123, 2014.

[19] L. Atzori, A. Iera, and G. Morabito, "The internet of things: A survey," Computer networks, vol. 54, no. 15, pp. 2787-2805, 2010. 